\title{
Medical Marijuana: A Hope For Resistant Epilepsy?
}

\author{
Arsalan Anwar, Sidra Saleem
}

\section{Dear sir}

Cannabis is a popular recreational drug worldwide and recently has been approved by many countries and a few states within the USA for recreational purposes. It is composed of two basic components, the psychoactive part tetrahydrocannabinol (THC) that targets CB1 and CB2 receptors in the body and counteracts reactive oxygen species and the Cannabinoidiol (CBD) component is famous for its medicinal use that works without interacting with these receptors and is free of psychiatric side effects.

$\mathrm{CBD}$ has gained wide popularity for its use such as reducing nausea/vomiting during chemotherapy, chronic pain, muscular spasm, improving appetite in chronic immunosuppressive disorders ${ }^{1}$. In the past, Cannabis has also been used for multiple CNS disorders including Parkinson Disease, Multiple Sclerosis, Alzheimer's Disease, Autism, Tourette syndrome, Huntington Disease, Neuropathic pain but the most prominent and earliest example is its usage for treatment of Epilepsy ${ }^{2}$.

The global prevalence of epilepsy is generally taken as between 5 and 10 cases per 1000 persons.Prevalence of epilepsy in general population in Pakistan is estimated to be 9.99 in 1,000 population. It is characterized by a history of at least one seizure, enduring alterations in brain with likelihood of future episodes. It can be due to different phenomenon such as Traumatic Brain Injury, Congenital Syndromes, Stroke and Infections. The other component is associated neurobiological, cognitive, psychological and social disturbances ${ }^{3}$. All these demographics is always concerning to researchers and they are actively working on Anti-Epileptic Drugs (AED) and therapeutics of all kind of Epilepsy.
Multiple treatment modalities are available for epilepsy but the advancement in treating resistant epilepsy has been slow. Cannabis has created hope in the epilepsy community for its modest efficacy in treating resistant epilepsy disorder (seizures cannot be controlled with the use of two appropriate AEDs). Resistant Epilepsy more commonly occurs in two congenital disorders-Dravet syndrome (DS) or LennoxGastaut syndrome (LGS) ${ }^{4}$. Multiple studies have been conducted in the past to assess the efficacy of Cannabis and showed improvement in symptoms but it is used as an adjunct with traditional AED in treating Epilepsy ${ }^{5}$. More studies are required before it will go mainstream.

\section{REFERENCES}

1. Borgelt LM, Franson KL, Nussbaum AM, Wang GS (February 2013). "The pharmacologic and clinical effects of medical cannabis". Pharmacotherapy(Review). 33 (2): 195-209.

2. Maroon J, Bost J. Review of the neurological benefits of phytocannabinoids. Surg Neurol Int. 2018;9:91. Published 2018 Apr 26. doi:10.4103/sni.sni_45_18

3. I. A. Khatri, S. T. Iannaccone, M. S. Ilyas, M. Abdullah, S. Saleem Epidemiology of epilepsy in Pakistan: review of literature J Pak Med Assoc. 2003; 53(12): 594-597.

4. O'Connell, B. K., Gloss, D., \& Devinsky, O. (2017). Cannabinoids in treatment-resistant epilepsy: a review. Epilepsy \& Behavior, 70, 341-348.

5. Zaheer, S., Kumar, D., Khan, M. T., Giyanwani, P. R., \& Kiran, F. N. U. Epilepsy and Cannabis: A Literature Review. Cureus, 2018; 10(9).

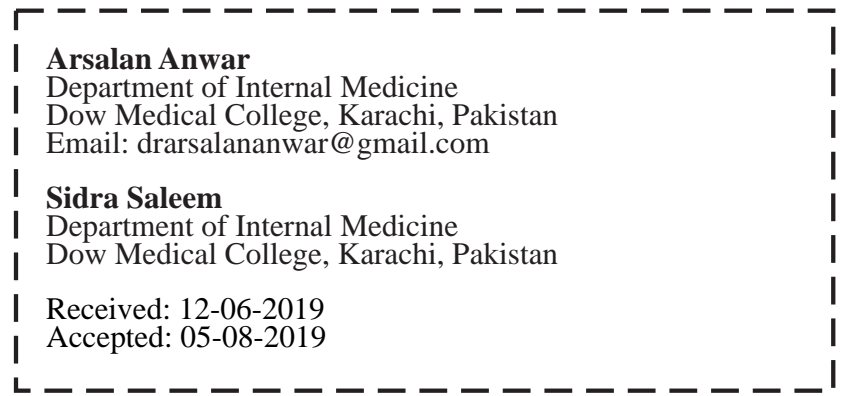

JBUMDC 2019; 9(4):326 